\title{
Flood forecasting model based on geographical information system
}

\author{
AN DONG ${ }^{\mathbf{1}}$, LI ZHI-JIA ${ }^{1}$, WU YONG-TUO ${ }^{2}$, YAO CHENG ${ }^{\mathbf{1}}$ \& DU YI-HENG \\ 1 College of Hydrology and Water Resources, Hohai University, Nanjing, 210098, China \\ andong hhu@outlook.com \\ 2 Shandong Electric Power Engineering Consulting Institute Corp. Ltd, Jinan, 250100, China
}

\begin{abstract}
In this paper, the Antecedent Precipitation Index Model (API) combined with Nash's Instantaneous Unit Curve Method is adopted for flood forecasting. The parameters $n$ and $k$ of Nash's Method is obtained by setting up the mathematic relation between these two parameters and topographic characteristics. Based on the DEM information, ArcGIS software is used to get the topographic characteristics and the topographic parameters. The Tunxi basin in the humid region was taken as an example for analysis. Through comparison with the simulation results of the Xinanjiang model, the detailed analysis of our simulation results is carried out giving a Nash-Sutcliffe efficiency 0.80 for the combined model and 0.94 for the Xinanjiang model. This indicates that the combined model as well as the Xinanjiang Model has a good performance in the simulation process. The combined model has great potential as a new efficient approach for flood forecasting in similar basins.
\end{abstract}

Key words antecedent precipitation index model; Nash's Instantaneous Unit Curve method; the geographic information system; Xinanjiang Model; Tunxi basin

\section{INTRODUCTION}

In recent decades, with the application of geographic information system (GIS) technology, developments in the field of hydrology and water resources have been promoted. The GIS has incomparable advantages for handling geographical and terrain data. It has become an important tool for flood forecasting models which play a vital role in flood control.

It is well known that both the Antecedent Precipitation Index model (API) and Xinanjiang model were widely used for flood forecasting in humid and semi-humid areas all over the world. The former model has a simple concept and at the same time low requirements for data, it is empirical and regarded as a 'black box model'. The latter one is a classical conceptual model with complete physical concepts and parameters. Their usefulness has been widely confirmed.

In this paper, the API model combined with Nash's Instantaneous Unit Curve Method is adopted for forecasting in Tunxi basin. Traditionally, the parameters of this model are mostly determined by trial and error, which brings inevitable error. By contrast, through the establishment of mathematical relationships between the landform parameters and Nash's Instantaneous Unit Curve Method parameters, $n$ and $k$, a more reliable method to determine the value of these two parameters is obtained by analysis of geographic information. This approach aims to avoid the blindness of the determination of the model parameters.

Besides, the Xinanjiang model has also been applied in the same basin. As a typical conceptual model, its simulation results are compared with the empirical combined model to explore the performance of different kinds of models in the basin.

Observations of 25 flood events from 1983 to 2001, in Tunxi basin which belongs to the humid region, were selected for simulation at the hourly time step.

\section{GEOGRAPHICAL INFORMATION-BASED FORECASTING METHOD}

\section{Antecedent precipitation index model}

The rainfall-runoff correlation diagram method is a conventional experimental method to study the rainfall-runoff problem. Based on the combination of cause analysis and statistical correlation, this method is a quantitative correlation diagram method which is established by using watershed average precipitation, corresponding runoff and the main influence factors of precipitation and runoff. It is also known as Antecedent Precipitation Index Model, usually abbreviated to API Model. 


\section{Nash's instantaneous unit curve}

To obtain the discharge curve of flood forecasting, the unit curve concentration calculation was applied to the runoff results of rainfall-runoff correlation. An Irish hydrologist, Nash, considered the flow process at the basin section as the result of regulation and storage of the net rainfall by the basin. From the view of the system, the concentration system of the basin is a linear, time invariant system, whose relationship of input and output can be described by $n$ order linear ordinary differential equations. And we can get the geomorphological instantaneous unit curve from Nash's instantaneous unit curve:

$$
\frac{(n-1) e^{(1-n)}}{(n-1) !}=0.58\left(\frac{R_{B}}{R_{A}}\right)^{0.55} R_{L}^{0.05}
$$

where $R_{B}, R_{L}$, and $R_{A}$ are the bifurcation ratio, stream-length ratio and area ratio, respectively, which can be calculated by Horton's law of river numbers, law of river length and law of area. Therefore, the parameter $n$ of the Nash model is a concentration parameter which mainly depends on the Horton parameters of the basin, and it mainly reflects the impacts of the basin area, the basin shape and river distribution on the flow concentration, which should not be simply interpreted as the number of linear reservoirs. The parameter $n$ can be obtained by using successive approximation to equation (1). Parameter $k$ is obtained from the topographic data, and determines the average concentration time according to the topographic data. After much research, Agnese et al. found that equation (2) can reflect the distribution of basin concentration time:

$$
\tau=1-(1-\lambda)(1-\rho)
$$

where $\lambda=\left(\sum_{j=1}^{n} \Delta l_{j}\right)\left(\sum_{j=1}^{J} \Delta l_{j}\right)^{-1} \rho=\left(\sum_{j=1}^{n}\left(\Delta l_{j} p_{j}{ }^{-0.5}\right)\right)\left(\sum_{j=1}^{J}\left(\Delta l_{j} p_{j}{ }^{-0.5}\right)\right)^{-1}$

and $\tau$ is the ratio of the average concentration time of a net rain particle from riverhead to a certain downstream section to the average concentration time from riverhead to basin export section; $\Delta l_{j}$ : the length of the first $j$ sub-reach divided from the riverhead; $p_{j}$ : the average slope of the first $j$ sub-reach; $j$ : the number of sub-reaches from the riverhead to the export section; $n$ : the number of sub-reaches from riverhead to the certain downstream section; $l$ : river length of the reach from riverhead to the given downstream section; $L$ : river length of the reach from riverhead to the export section.

Further analysis gives:

$$
\tau=\lambda^{1-m \lambda}
$$

where, $m$ is a comprehensive parameter which reflects the longitudinal slope features of the river.

As long as the flow velocity of the export section of the river basin is provided, the parameter $k$ can be obtained according to:

$$
\mathbf{k}=\frac{\alpha L_{\Omega}}{n\left(\mathbf{1}-\lambda_{\Omega-1}^{1-m \lambda_{\Omega-1}}\right)} v_{\Omega}^{-1}
$$

where $\Omega$ is the Strahler of river basin level, that is the highest level of the river system; $L_{\Omega}$ is the length of the river with highest level in the river system; $\lambda_{\Omega-1}$ is the value of $\lambda$ from the riverhead to the end of river with $\Omega-1$ level; $v_{\Omega}$ is the flow velocity of the export section of the river basin, generally given by the average velocity of the rise of the flood hydrograph at the export section; $\alpha$ is the ratio of distance from the watershed centroid to the export section of the river basin, to the basin length.

From equation (4), parameter $k$ is determined by the length of river with highest level, slope parameters, and the velocity, meanwhile the velocity is also associated with slope, so the parameter $k$ can be seen as a hydrodynamic diffusion coefficient. 


\section{The determination of parameters $\boldsymbol{n}$ and $\boldsymbol{k}$}

The Tunxi catchment is located in southeast China with a drainage area of $2696.76 \mathrm{~km}^{2}$. The altitude increases gradually from the east to the west; there is a large area of forest land in the upstream region. The average annual temperature is $17^{\circ} \mathrm{C}$. The spatial distribution of rainfall varies widely from year to year, as affected by the continental monsoon climate. The annual rainfall increases with the elevation; the rainfall in the mountainous area is more than that in the plain area. Moreover, the annual rainfall varies significantly. In each year, the rainfall amount from April to June is more than $50 \%$ of the annual precipitation.

Statistics of the topographic parameters of Tunxi basin can be obtained through processing of the DEM data (Table 1). Substituting those parameters into equation (1) gave the value of parameter $n$ using the successive approximation method, and the value is 3.069.

Table 1 Topography parameters of Tunxi basin.

\begin{tabular}{rrrlcrlr}
\hline $\begin{array}{l}\text { River } \\
\text { level }\end{array}$ & $\begin{array}{l}\text { River } \\
\text { number }\end{array}$ & $\begin{array}{l}\text { Average } \\
\text { river length } \\
(\mathrm{km})\end{array}$ & $\begin{array}{l}\text { Concentration } \\
\text { area }\left(\mathrm{km}^{2}\right)\end{array}$ & $\begin{array}{l}\text { Average } \\
\text { concentration area } \\
\left(\mathrm{km}^{2}\right)\end{array}$ & $R_{B}$ & $R_{L}$ & $R_{A}$ \\
\hline 1 & 120 & 4.043 & 1757.3921 & 14.645 & 5.038 & 2.366 & 5.975 \\
2 & 26 & 9.429 & 1763.5391 & 67.828 & & & \\
3 & 4 & 39.983 & 2373.2837 & 593.321 & & \\
4 & 1 & 21.023 & 2696.7674 & 2696.767 & & \\
\hline
\end{tabular}

With three typical rivers, the relationship of river slope $p_{j}$ and river length delta $\Delta l_{j}$ were respectively extracted, and $\tau$ and $\lambda$ calculated for each river according to equation (2). $\tau-\lambda$ diagrams for the three river ( $\tau$ 's value as the horizontal axis and $\lambda$ 's value as the vertical axis) were produced, and finally the value of $m$ of 0.9 obtained by the curve fitting method.

As $\alpha$ is the ratio of distance from watershed centroid to the export section of the river basin to the basin length, the value of $\alpha$ is approximately equal to 0.386 by calculation:

$$
v_{\Omega}=\frac{\sum_{i=0}^{p-1}\left(\frac{v_{i}-v_{i+1}}{2}\right)\left(t_{i+1}-t_{i}\right)}{t_{p}-t_{0}}
$$

where $t_{i}$ is the moment of time $i$ of the rising flood process; $v_{i}$ is the velocity of the rising flood process at time $t_{i} ; t_{p}$ is the moment of flood peak; $t_{0}$ is the moment when river begins to rise.

Substitute the values of $m, \alpha, v_{\Omega}, \lambda_{\Omega-1}$ and $L_{\Omega}$ into equation (4) to obtain the value of parameter $k$ of 6 , so the instantaneous unit curve can be worked out.

\section{XINANJIANG MODEL}

As a conceptual model, the Xinanjiang Model has been widely applied in flood forecasting in China, and good application results have been obtained. When using the Xinanjiang Model for hydrological simulation, first the basin should be divided into several units according to the rainfall and underlying surface characteristics, then hydrological calculations should be taken for each unit to get the flow process of each unit. Finally, calculus and superposition should be performed to the export to get the flow process of the whole basin. The model is composed of four modules, namely modules of evapotranspiration, runoff generation, water source division, and flow concentration, and each module has correspondingly different model parameters.

\section{THE SIMULATION RESULT OF TWO MODELS}

The Antecedent Precipitation Index Model is a kind of empirical method. The relationship of precipitation and runoff is established on the correlogram. This kind of empirical correlation relation to some extent is guided by physical concepts, but lacks rigour and integrity. And Nash's Instantaneous Unit Curve Method is a kind of logical method without any physical concept. The 
combination of the API and Unit Curve leads to a simple structure for flood forecasting and it has a quite good performance for some basins. But this method has some difficulties to be improved due to the lack of physical significance.

The Xinanjiang Model has a specific physical structure and meaning, and also 15 different parameters. The applicability of the model is very strong and because of its clear modules, it is easy to adjust the structure to adapt to a certain basin's characteristics. However, the weakness of the Xinanjiang Model is the problem of uncertainty, the complexity of parameter calibration, and the performance is not always better than a black box model for a same basin. In particular, like some other conceptual models the Xinanjiang Model could just fit for come specific basins.

The above-mentioned models are taken for flood simulation on Tunxi basin. The simulation results of API combined with Nash's Instantaneous Unit Curve Method and the Xinanjiang Model are given in Table 2 (API stands for combined model, XAJ for Xinanjiang Model). The assessment criteria used are the relative runoff error (RRE, \%), relative peak error (RPE, \%), peak time error (PTE, hour) and Nash-Sutcliffe efficiency (NSE).

Table 2 Flood simulation characteristic value of API and XAJ model on Tunxi basin.

\begin{tabular}{|c|c|c|c|c|c|c|c|c|}
\hline \multirow{2}{*}{$\begin{array}{l}\text { Start time of } \\
\text { each flood }\end{array}$} & \multicolumn{2}{|c|}{ RRE (\%) } & \multicolumn{2}{|c|}{ RPE (\%) } & \multicolumn{2}{|c|}{ PTE } & \multicolumn{2}{|c|}{ NSE } \\
\hline & API & XAJ & API & XAJ & API & $\mathrm{XAJ}$ & API & XAJ \\
\hline 1983051108 & 3.8 & 8.79 & 5.8 & 1.8 & -1 & -1 & 0.95 & 0.95 \\
\hline 1983051422 & 5.2 & -15.08 & -10 & -9.5 & -4 & -1 & 0.95 & 0.93 \\
\hline 1984060502 & 21.2 & 3.2 & 31 & -6.3 & -4 & 0 & 0.7 & 0.98 \\
\hline 1984083008 & 4.8 & -7.2 & 17.9 & 5.5 & 0 & 5 & 0.94 & 0.98 \\
\hline 1986061108 & -15.5 & 7.35 & -2.1 & -3.3 & 0 & -1 & 0.63 & 0.95 \\
\hline 1987050108 & -5 & 13.19 & -13.3 & 26.7 & 0 & -1 & 0.86 & 0.81 \\
\hline 1988050704 & 19.8 & 11.28 & 30.8 & -5.8 & -3 & 0 & 0.83 & 0.94 \\
\hline 1988061101 & 15.2 & 2.44 & 5.1 & -13 & -1 & 0 & 0.76 & 0.9 \\
\hline 1989061206 & 12.7 & -2.46 & 20.7 & 0.7 & -3 & -1 & 0.91 & 0.98 \\
\hline 1989063023 & 9.3 & -2.57 & 28.6 & -13.8 & -4 & -1 & 0.83 & 0.96 \\
\hline 1990061108 & -7.9 & -7.85 & -0.6 & 6 & 2 & -4 & 0.69 & 0.94 \\
\hline 1991063008 & 25 & 2.3 & 42 & 25.4 & -3 & 1 & 0.71 & 0.93 \\
\hline 1991072508 & -16.1 & -5.7 & -4.7 & 34.1 & -5 & -3 & 0.56 & 0.9 \\
\hline 1992062000 & -8.3 & -7.94 & -2.8 & 0.7 & -3 & 0 & 0.85 & 0.97 \\
\hline 1993052700 & -12.1 & 3.52 & -23.6 & 19.7 & -2 & -1 & 0.77 & 0.97 \\
\hline 1994050100 & -12.6 & -3.61 & -17.8 & 3 & -2 & -1 & 0.86 & 0.98 \\
\hline 1995051500 & -5.5 & -1.19 & 7.4 & -4.3 & -4 & 1 & 0.76 & 0.94 \\
\hline 1996060100 & -10.2 & 3.23 & 11.6 & -0.5 & 0 & -3 & 0.92 & 0.98 \\
\hline 1997070208 & -6 & -20.8 & 3.9 & -9.8 & -4 & -2 & 0.78 & 0.95 \\
\hline 1998050108 & -14.9 & -0.7 & 5.5 & -9.5 & -6 & 0 & 0.82 & 0.97 \\
\hline 1999062215 & 7.6 & -10.1 & -0.1 & -8.4 & 1 & -2 & 0.92 & 0.95 \\
\hline 1999082408 & -2.7 & -0.6 & -2.6 & 19.5 & -4 & -1 & 0.8 & 0.95 \\
\hline 2001050108 & -16.8 & -5.47 & -13 & 11.3 & -3 & -1 & 0.73 & 0.91 \\
\hline 2001062008 & 17.3 & 8.7 & 26.4 & 14.5 & -8 & 5 & 0.78 & 0.93 \\
\hline 2001070908 & -12.4 & -22.2 & -12.9 & -20.1 & 1 & 2 & 0.74 & 0.84 \\
\hline Average value & -0.2 & -2.0 & 5.3 & 2.6 & & & 0.80 & 0.94 \\
\hline
\end{tabular}


The flood simulation characteristic values (Table 2) for the combined model show the qualification ratio of runoff simulation is $92 \%$, and for the ratio of peak simulation is $72 \%$, and the average value of NSE is 0.8 , which is a good simulation result. For the Xinanjiang Model, the qualification ratio of runoff simulation is $92 \%$, the qualification ratio of peak simulation is $84 \%$, and the NSE average is 0.94 , i.e. the Xinanjiang Model did even better. The qualification ratios of runoff simulation are both more than $80 \%$ and at the same value. For the rest of the results, the Xinanjiang Model is more reliable.

\section{CONCLUSION AND PROSPECTS}

This paper introduced the Antecedent Precipitation Index Model (API) combined with Nash's Instantaneous Unit Curve Method for flood simulation and the determination method of Nash's Instantaneous Unit Curve parameters $n$ and $k$ through the analysis of geographic information. The research takes Tunxi basin in humid area as an example to validate the practical utility of combined model.

The performances of the API combined with Nash's instantaneous unit curve method and the Xinanjiang Model were compared. Both of the simulation results are accurate and stable, especially the Xinanjiang model. This reconfirms again the good forecasting ability of the Xinanjiang Model in humid regions. We can see from the result that the combined model is not the best one for Tunxi basin, but it does not mean it should be replaced by the Xinanjiang Model. It has a simpler structure and fewer parameters. It is more convenient for application, especially in regions without long-term hydrologic data because of the low data-demand compared to the Xinanjiang Model. On the basis of keeping all the advantages of the combined model, the determination method of the parameters through analysing the geographical information is introduced in this research. It brings physical meaning to the parameters of Nash's Instantaneous Unit Curve Method.

Notwithstanding the good performance of both models, there are still some shortcomings in the research work:

(a) The integrity of the hydrological data is the precondition of hydrologic forecasting model, there still is a problem of some missing data.

(b) Sensitivity analysis of the model parameters and the uncertainty analysis in the process of hydrological simulation are necessary to include in the research process.

Acknowledgements This work was supported by the National Natural Science Foundation of China (Grant Nos. 41130639, 41101017 and 51179045).

\section{REFERENCES}

Georgakakos, K. (2006) Analytical results for operational flash flood guidance. Journal of Hydrology 317, 81-103.

Strahler, A. (1957) Quantitative analysis of watershed geomorphology. Trans. Am. Geophys. Union 38(6), 913-920.

Agnese, C., et al. (1988) Estimation of the time scale of the Geomorphologic Instantaneous Unit Hydrograph from effective streamflow velocity. Water Resources Research 24(7), 969-978.

Shi, P., et al. (2003) Determination of parameters for Nash runoff model by DEM. Journal of Hohai University: Natural Sciences 31(4), 378-381.

Yao, C., et al. (2012) Parameter estimation and application of grid-based Xinanjiang Model. Journal of Hohai University: Natural Sciences 40(1), 42-47.

Zhao, R.J. (1984) Watershed Hydrologic Modeling. Beijing: Water Resources and Electric Power Press.

Zhao, R.J., et al. (1995) The Xinanjiang Model. Colorado: Water Resources Publications. 